\title{
Order Parameter Re-Mapping Algorithm for 3D Phase Field Model of Grain Growth Using FEM
}

\author{
Cody J Permann ${ }^{\mathrm{a}}$, Michael R Tonks ${ }^{\mathrm{b}}$, Bradley Fromm ${ }^{\mathrm{b}}$, Derek R Gaston ${ }^{\mathrm{a}}$ \\ ${ }^{a}$ Modeling and Simulation, Idaho National Laboratory, P.O. Box 1625, Idaho Falls, ID 83415. \\ ${ }^{b}$ Fuel Modeling and Simulation, Idaho National Laboratory, P.O. Box 1625, Idaho Falls, ID 83415.
}

\begin{abstract}
Phase field modeling (PFM) is a well-known technique for simulating microstructural evolution. To model grain growth using PFM, typically each grain is assigned a unique non-conserved order parameter and each order parameter field is evolved in time. Traditional approaches using a one-to-one mapping of grains to order parameters present a challenge when modeling large numbers of grains due to the computational expense of using many order parameters. This problem is exacerbated when using an implicit finite element method (FEM), as the global matrix size is proportional to the number of order parameters. While previous work has developed methods to reduce the number of required variables and thus computational complexity and run time, none of the existing approaches can be applied for an implicit FEM implementation of PFM. Here, we present a modular, dynamic, scalable reassignment algorithm suitable for use in such a system. Polycrystal modeling with grain growth and stress require careful tracking of each grain's position and orientation which is lost when using a reduced order parameter set. The method presented in this paper maintains a unique ID for each grain even after reassignment, to allow the PFM to be tightly coupled to calculations of the stress throughout the polycrystal. Implementation details and comparative results of our approach are presented.
\end{abstract}

Keywords: PF method, Finite Element, Grain Growth, 3D Modeling, MOOSE

\section{Introduction}

Grain growth and grain boundary (GB) migration are critical phenomena in material engineering and design and have been modeled for many years. Grain boundary motion is driven by reduction of overall free energy in the system. Driving forces for GB migration include the reduction in GB, deformation and defect energy. The earliest grain growth models were Monte Carlo Potts models [1, 2], but since then researchers have employed front tracking $[3,4]$, phase field $(\mathrm{PF})[5,6]$, cellular automata [7], and level set methods [8]. These methods were first developed to model grain boundary migration driven by the reduction of GB energy, but many have been expanded to consider the reduction of other sources of energy [9-13]. While all of the various models have been shown to predict similar behavior, Monte Carlo Potts models and the phasefield method are the most popular due to their flexibility and computational efficiency.
In the PF method, grain growth is typically modeled by representing each grain by an order parameter $\phi_{i}[5,6]$, where $\phi_{i}=1$ within its corresponding grain, $\phi_{i}=0$ within all other grains and $0<\phi_{i}<1$ within grain boundaries. The diffuse interface is not tracked explicitly, it is instead defined for a range of values in $\phi$. This approach has been used effectively in $2 \mathrm{D}$ and $3 \mathrm{D}$ and has been quantified for specific materials [14]. It has been solved using finite difference, spectral methods using the fast Fourier transform and the finite element method (FEM), though spectral methods are the most common.

Each of these solution methods have strengths and weaknesses. The strengths of FEM are that it can model a large range of domain shapes and boundary conditions, and it allows easy coupling of the PF equations to other physics, such as mechanics or heat conduction. In addition, FEM can be run in parralel on many processors with excellent scalability. Implicit FEM adds the benefit of being able to maintain stability with large time steps. 
The primary weakness is long solve times due to computational expense, which can be reduced by parallel computing, as well as by mesh and time step adaptivity.

A major limitation of PFM is the large computational cost when modeling polycrystals with many grains due to the large number of required variables. However, though one variable is used for each grain, at any point in the domain only a few of the variables have nonzero values. Thus, various researchers have developed methods that take advantage of this fact to drastically reduce the computational cost. In Krill and Chen [15], order parameters were used to model multiple grains; once two grains represented by the same order parameter came close to touching, one grain was reassigned to a new order parameter. This approach required a minimum of 17 order parameters in $2 \mathrm{D}$ and 25 in 3D. Also, grains did not have a unique ID that could be used for assigning properties such as the grain orientation. Kim et al. [6], Gruber et al. [16], Vedantam and Patnaik [17] employed a unique order parameter for each grain, but used sparse data structures to locally store only order parameters that were nonzero or were nonzero at adjoining points. This approach is more computationally efficient than that from Krill and Chen [15] and it has a unique ID for every grain. While the previous methods were used for explicit time integration, Vanherpe et al. [18] developed a bounding box algorithm that worked for semi-implicit time integration schemes. None of the methods mentioned here are suitable for a fully-implicit FEM solution.

The Multiphysics Object Oriented Simulation Environment MOOSE is a framework for solving complex PDEs. It has been used to develop a wide range of multiphysics models [19, 20]. The Phase Field Module within the MOOSE framework provides tools to quickly develop PF models coupled to other physics such as mechanics or heat conduction [21]. It solves the fully-coupled multiphysics equations using FEM and fully implicit time integration. The PF grain growth evolution model has been implemented in the code, but it is computationally expensive, as none of the existing efficient algorithms can be used with fully-implicit FEM.

In this work, we present an efficient algorithm for the PF grain growth model that uses grain remapping, and can be used with FEM and implicit time integration. In addition, the algorithm is parallel, works with various boundary conditions and main- tains a unique ID of each grain that can be used for applying material properties or tracking individual crystallographic grain orientation within a simulation. In this work, we first summarize the implementation of the algorithm. We then verify its predictions by comparing results of non-reduced (number of order parameters equal to the number of grains) simulations with reduced (number of order parameters less than the number of grains) simulations employing the grain tracking algorithm. Finally, we demonstrate the algorithm in two and three dimensions and characterize its performance.

\section{Implementation}

The grain tracking algorithm is actually the combination of two algorithms: a flood algorithm that identifies the individual grains and a grain tracking algorithm that tracks movement, detects contact, and remaps grains when necessary. The two algorithms are combined and run as a post-processing step after the nonlinear solve for each time step has converged, and therefore do not impact the nonlinear solves required for the implicit solution. In addition, they are compatible with mesh adaptivity and parallel computation. Finally, the grain tracker is also object-oriented and designed for easy extensibility for use in new application models.

\subsection{Flood Algorithm}

The first algorithm identifies grain volumes by using a standard stack-based recursive flood fill routine [22] for each of the order parameter fields. Each arbitrary grain volume is completely identified and "colored" by the flood fill defined by the enclosing grain boundaries. The grain tracker has been designed to work with two different mesh entity types: nodes or elements. While the choice of entity type doesn't impact the capabilities of the grain tracker, the information that can be retrieved through the grain tracker interface changes depending on the chosen entity type. For example, when running the grain tracker in "element" entity mode, one can query the grain tracker object for what grain is active for a given element. When running the grain tracker in "node" entity mode, one can query the grain tracker object for what grain is active for a given node.

To introduce the operation of the flood routine, we must first illustrate the mesh decomposition used in the simulation. MOOSE makes use of 


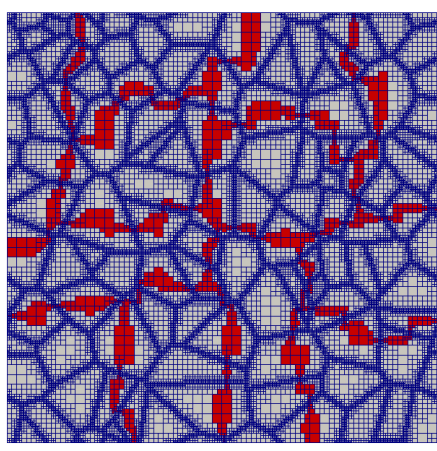

Figure 1: A 12-way partitioned mesh with ghosted elements shaded. The "semi-local region" for an individual processor is an outlined region including the shaded elements immediately surrounding that region. This mesh is adapted to the grain boundary field so the finest elements appear on and near grain boundaries while the coarsest elements appear in grain interiors. Note: Several grains are split over processor boundaries.

the partitioning given by the underlying library, libMesh [23]. Each processor is given a balanced non-overlapping region of elements to process called the "local region". An additional region of "ghost" elements is maintained along the border of these partitions to facilitate information exchange with neighboring processors. The local region combined with the ghost region is called the "semi-local region" (Fig. 1).

The algorithm begins with each processor looping over all of the elements within its local region. A call is made to the flood routine for appropriate entity type (either the element itself, or each of the nodes on the element). Inside the flood routine the solution values of each of the order parameters are available for inspection for the current entity. If any $\phi_{i}$ is above the chosen threshold identifying a region of interest, that entity is marked with a processor local unique number. The flood routine then recursively visits all connected neighbors of the current entity type marking them with the same number if the corresponding $\phi_{i}$ variable values are also above the desired threshold. The recursion terminates if any one of three criteria are met: a value is encountered that falls below the desired threshold, a previously marked entity is re-visited, or a non-local entity (not in the semi-local range) is encountered. It is important to note that these marked regions do not correspond to grains at this stage due to mesh decomposition; we'll refer to them simply as "partial features" or "locally connected regions". Each order parameter's flood markings are maintained in separate data structures so that connected regions of each order parameter can overlap slightly in the grain boundary regions. These independent data structures allow for arbitrary overlap defined by the variable value threshold and are necessary for later stages of the algorithm.

After the flood algorithm has produced a series of processor local connected regions, that information is gathered and shared among all processors to produce "globally connected regions" or "grain maps" (one map for each order parameter in the simulation). A data marshaling (also known as serialization) routine packs up the entity numbers and corresponding order parameter numbers into a single long vector for efficient parallel communication. These vectors are then replicated on every processor through a global communication. Global communication is necessary as grains can grow arbitrarily large and can take on arbitrary shapes spanning several processor boundaries in the domain. Grains may be partially represented on several processors in a parallel job. After the data has been replicated on all processors it is unmarshalled through a reverse process into local data structures for use by the remaining stages of the algorithm. The next stage of turning these marked entities into a global grain map consists of merging together partial connected regions along processor boundaries where mesh entity information overlaps. This merging or "stitching" is possible thanks to the use of the "semi-local" element ranges used during the flood stage. Any time duplicate entity information is encountered between two or more connected regions, those regions are merged into a single larger region until no more overlapping regions are present. At this point each processor contains a complete global grain map.

\subsection{Grain Tracking Algorithm}

Once the flood algorithm has finished, the grain tracking algorithm is executed. The grain tracking algorithm has three main purposes; track grain evolution across time steps while maintaining unique IDs, detect impending contact between grains, and remap grains to new order parameters to avoid grain coalescence. Unintentional grain coalescence will occur in the PF model when two grains represented by the same order parameter become neighbors, as the equations cannot distinguish them. Grain coalescence is non-physical behavior and 
must be avoided to correctly model physical microstructure evolution with a reduced order parameter set. Note that this problem does not occur when each order parameter represents a single unique grain.

To begin, the grain tracking algorithm must compute a globally consistent picture of the microstructure with the grain map produced by the flood algorithm. The unique ID capability of the grain tracker requires that each grain from the current time step be matched up with the corresponding grain from the previous time step taking into account GB migration and the shrinking of grains until they disappear. This process begins by taking the grain maps (described in Section 2.1) and constructing a simpler geometric description of the microstructure. Each grain which may be of arbitrary shape and size is approximated by a sphere enclosing the entire region. An additional buffer zone may also be added to each sphere which is useful during the collision detection phase. If periodic boundaries are used in the simulation, grains intersecting any of the domain edges that are on a periodic boundary are partially represented at multiple disjoint locations on the Cartesian domain. To handle these disjoint regions, each portion of the grain is represented as a separate enclosing sphere. In a fully periodic domain in three dimensions a single grain on the corner can be represented by up to eight independent spheres. The simplified geometry gives a complete picture of the microstructure for use in the tracking and remapping stages. For full generality, the simplified geometry consists of several enclosing spheres, each which can be described by a centroid and a radius.

The tracking stage begins after the simplified geometry is constructed. Each grain's simplified geometry description from the previous time step is used to locate the corresponding unassigned grain region in the current time step. This is done by selecting the grain with the closest centroid with a matching order parameter for every grain. This algorithm is guaranteed to succeed given a valid order parameter configuration at each time step. This is due to the fact that grains represented by a single order parameter are spaced such that their simplified geometries do not touch, thus adjacent neighboring grains will therefore always have a different order parameter representation ensuring that minimizing the the centroid movement will not incorrectly identify a given grain with an assigned order parameter due to grain evolution. Finally each unique grain contains a status variable indicating whether the grain is active, or inactive meaning that the grain has been absorbed.

Dealing with disappearing grains using this algorithm is also straightforward. If a grain disappears in the current time step, the algorithm will incorrectly select another nearby grain with a matching order parameter as the new location. This action results in some grains being claimed by multiple grains from the previous time step leading to a conflict. To resolve this conflict, a list of all grains claiming ownership of the current time step's grains is maintained until all grains have selected their closest matches. Only the closest grain will be allowed to continue on to the next time step, while all of the remaining grain's status indicators will be marked as "inactive". The closest grain is always the correct match since all other choices would represent a grain being in contact with the closest grain which is an invalid configuration.

The same centroid movement minimization algorithm is used for grains spanning periodic boundaries. The minimum centroid distance is calculated simply as the minimum distance from any representative sphere to any other representative sphere of a given grain. This avoids large jumps in minimum distances as grains migrate towards or away from any periodic edge in the simulation. In any time step where a grain either enters or exits a periodic boundary at least one existing bounding sphere will remain close to one or more of the centroids in the previous time step. At the end of the tracking stage, all grains will have selected updated regions resulting in updates to the simplified geometry of the previous time step while maintaining their original uniquely assigned IDs. Any number of grains may be marked as inactive in any given step.

\subsubsection{Remapping Stage}

After the tracking stage has completed, remapping begins. The simplified geometry of each grain is compared to the simplified geometry of all other grains represented by the same order parameter. Any edge contact or overlap in any of the bounding spheres will trigger a remap operation as illustrated in Fig. 2(a). In the description of the remapping operation, we will refer to the grain being remapped as the "source" grain, and refer to the candidate order parameters (all others) as the "destination variables". When contact or intersection occurs, the list of grains represented by the destination variables are checked to locate a suitable order param- 
eter where the source grain can be remapped. The criterion for this selection is straightforward. The distance between the source sphere surface and the closest grain in each of the destination variables is constructed and ranked by distance. An attempt to remap the target grain will be made to one of the top variables in list order. Unfortunately this implementation is not guaranteed to find a suitable remapping candidate as this approach amounts to a greedy coloring algorithm [24]. In other words, it may not be possible to remap a single grain or shift multiple grains to satisfy the non-touching spheres requirement. A more sophisticated map-coloring algorithm to find a valid configuration may be required to satisfy every step given a valid initial condition. Future plans for the remapping algorithm are discussed further in Section 4.

Once a suitable candidate is found, the solution values defining the grain are copied from the source order parameter solution to the destination order parameter solution, as shown in Fig. 2(b). Additionally the order parameter stored in the target grain is updated in the unique grains data structure to reflect the change for future tracking steps. If no suitable order parameter is found for remapping, due to an insufficient number of order parameters, the current remapping operation is aborted and the simulation aborts. This avoids incorrect behavior of the simulation if the grain tracker is unable to perform its function.

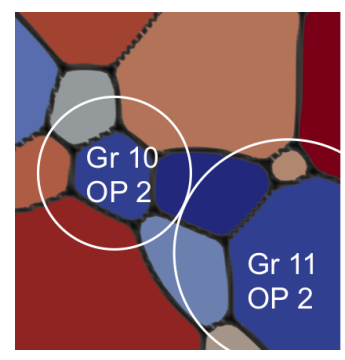

(a)

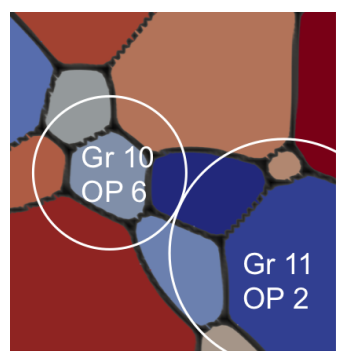

(b)
Figure 2: Demonstration of the remapping of grains, where (a) shows the bounding spheres of two grains (Gr) represented by the same order parameter (OP) coming in contact and (b) shows the remapping of one of the grains to a different order parameter. The grains are shaded by the order parameter number being used to represent them. The bounding spheres are constructed using the order parameter threshold value, here set to 0.05 , and therefore extend past the GB.

\subsection{Capability Summary}

The grain tracking algorithm provides the capability for multiphysics polycrystal simulations containing thousands of grains to be represented with a much smaller number of order parameters while using a fully-implicit time integration scheme. In addition, each grain has a unique ID throughout the simulation that can be tied to properties such as the crystallographic grain orientation. Several other grain attributes are available including the number of grains and the volume of every grain throughout the simulation. This capability is a modular design that can be added or removed from any phase field grain growth simulation without changes to the phase field equations. The grain tracker's additional information can be used for visualization of the grain structure or for tracking unique orientation. It can display fields indicating which order parameters own which grains, grain centroids, or even bounding spheres. In lieu of a visualization tool, field data can be output in any number of ASCII formats or queried at specific points within the domain on demand. Finally, all of these capabilities are easily extendable for additional visualization or future research.

\section{Results}

We investigate the performance of the grain remapping algorithm using various $2 \mathrm{D}$ and $3 \mathrm{D}$ simulations. Every simulation is run in parallel on at least 24 processors using a 16,000 core cluster at Idaho National Laboratory.

\subsection{Grain growth in a $2 D$ copper polycrystal using periodic boundary conditions}

We model a 2D copper polycrystal on a $1 \mu \mathrm{m} \times$ $1 \mu \mathrm{m}$ domain with 450 initial grains. The grain growth is predicted using the model from Moelans et al. [14] and implemented using the MOOSE Phase Field module. The initial grain structure is created using a Voronoi tessellation and the simulation is run at $T=500 \mathrm{~K}$ until 25 grains remain (see Figs. 3(a) and 3(b)). To represent copper, we assume isotropic GB properties and use a grain boundary mobility of $M_{G B}=2.5 \times$ $10^{-6} e^{-0.23 / k_{b} T} \mathrm{~m}^{4} /(\mathrm{Js})$ and GB energy $\gamma_{G B}=$ 0.708 taken from Schönfelder et al. [25] for a $\Sigma 29$ twist boundary. The solution was computed using implicit backward-Euler time integration with periodic boundary conditions. The simulations utilized 
mesh and time step adaptivity, to reduce computational expense.

450 grains were represented within the grain tracker using various numbers of order parameters ranging from 10 to 30 in increments of two. To evaluate the impact of the number of order parameters on the simulation, we plotted the average grain area over time (Fig. 3(c)). Simulations with less than 14 order parameters failed due to an insufficient number of order parameters for remapping. Simulations with 16 or more order parameters predicted the same grain growth irrespective of the number of order parameters used. The run containing 14 parameters failed to predict the same microstructure evolution due to an invalid initial condition where adjacent grains where assigned to the same order parameter. The average grain area over time is also shown for a simulation with 16 order parameters run without the remapping algorithm. The increase in the average grain area is significantly faster due to coalescence which is non-physical for this model.

A valid initial assignment of the order parameters is necessary to avoid coalescence during the first time step. If the initial condition assigns two adjacent grains to the same order parameter, a comparative study cannot be performed with that run since grain coalescence occurs in the initial time step causing different microstructural evolution. This deficiency and potential solutions are discussed further in Section 4. This is not a defect of the grain tracker algorithm.

The grain tracker algorithm increases the computational expense when compared to running the same reduced order parameter model without the grain tracker but guarantees the same behavior of a simulation running on a non-reduced order parameter set (correct evolution without coalescence). In the 2D 450 grain simulations, the grain tracker used 8 percent of the total computation time for the 30 order parameter simulation and approximately 11 percent of the computation for the 16 order parameter simulation. However when comparing to a nonreduced $2 \mathrm{D}$ simulation on the same microstructure, the savings is substantial in terms of both memory and time. Typical simulations run about two orders of magnitude faster and consume roughly two orders of magnitude less memory.

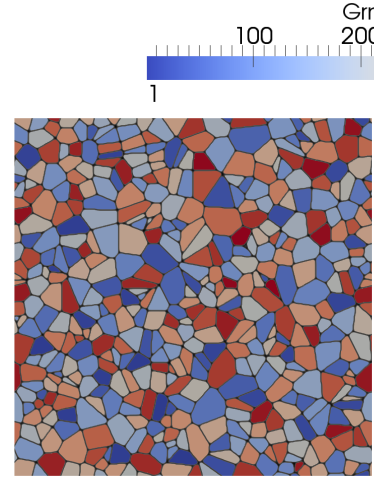

(a)
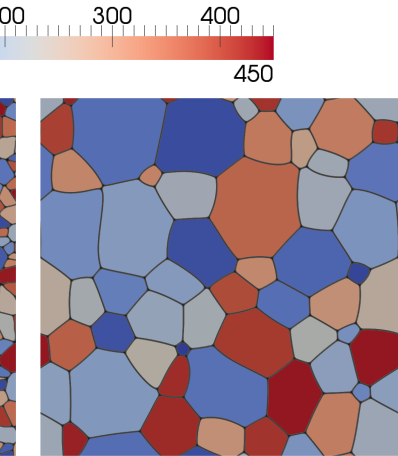

(b)

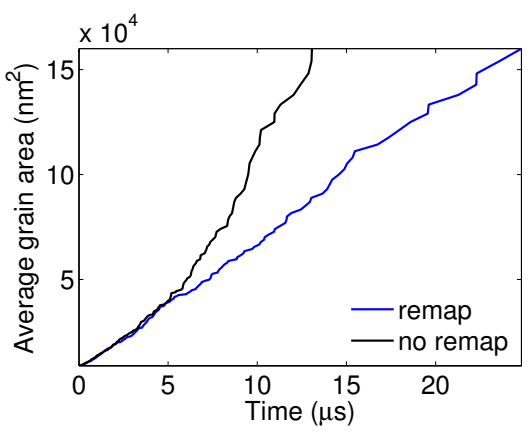

(c)

Figure 3: 2D simulation with grains spanning periodic boundary conditions. (a) initial grain structure, (b) final grain structure after $6000 \mathrm{~ns}$, (c) plot of the average grain area versus time. (a) and (b) are shaded by unique grain ID. Average grain area behaves the same for sixteen or more order parameters once the grain tracker is activated. Therefore, the model is evolving correctly (without coalescence).

\subsection{Grain growth in a circular $2 D$ copper polycrys- tal}

The grain tracker algorithm works for all domain sizes and shapes. In this example, we model grain growth in a circular polycrystal with 1735 initial grains, where the initial grain structure was randomly generated (see Fig. 4(a)). The simulation was conducted with 17 order parameters. Again, we simulated the grain growth at $T=500 \mathrm{~K}$ and the same model and material parameters were used as were used in the previous example. The domain has a $500 \mathrm{~nm}$ radius and zero flux boundary conditions were applied to all order parameters on the outer edge.

After $1.23 \mu \mathrm{s}, 111$ grains remain in the system, as shown in Fig. 4(b). The average grain area over time can be computed from the number of grains recorded by the grain tracker algorithm, showing 
the expected linear relationship (Fig. 4(c)). The grain tracker also records the grain area, from which we calculated the grain size distribution. The size distribution quickly reaches a steady state value, as shown in Fig. 4(d). The Hillert distribution [26] is shown for reference. The grain tracker algorithm took $11.96 \%$ of the total computation time.

\subsection{Grain growth in a deformed 2D copper poly- crystal}

Because the grain tracker algorithm maintains a unique ID for every grain throughout the simulation, it can be used with models that consider the impact of crystal orientation. In this simulation we model grain growth in a $2 \mathrm{D}$ copper polycrystal that is undergoing deformation using the model from Tonks et al. [12] and Tonks and Millett [13]. The polycrystal has 400 grains with the initial structure created by a Voronoi tessellation, as shown in Fig. 5(a). We assume isotropic GB properties, though we consider elastic anisotropy. The initial texture is random and only involves rotations around the $z$-axis ranging from $0^{\circ}$ to $45^{\circ}$, due to the cubic symmetry. Sixteen order parameters were used to represent the 400 grains.

To account for the mechanical deformation, we couple the phase field equations to a solution of a linear elastic mechanics problem, in the manner shown in Tonks et al. [21]. The values for the three cubic elastic constants for copper are $C_{11}=$, $C_{12}=$, and $C_{14}=[25]$. The square domain is $1 \mu m \times 1 \mu m$ and it is deformed downward on the top boundary by 50 and $100 \mathrm{~nm}$, in two simulations respectively. The applied deformation begins at zero at time equals zero and increases linearly until it reaches the maximum value at $t=25 \mathrm{~ns}$. For the rest of the simulation, the applied deformation is constant. The bottom surface is fixed in the $y$-direction, while the left and right boundaries are fixed in the $x$-direction. Again, the simulation is solved using implicit time integration and mesh and time step adaptivity to reduce the computational expense. The grain tracker algorithm took only $2.8 \%$ of the total computation time, due to additional costs from the mechanics calculation.

As shown in the previous work [13], the slope of the plot of grain area over time increases with increasing magnitude of the applied deformation (see Fig. 4(d)). As the grains grow under the applied load, specific orientations grow preferentially over other grains in order to reduce the total elastic energy in the system. With no applied load, the final texture is random. As the applied load increases, the final texture becomes more and more pronounced (Figs. 5(b) to 5(d)). Note that this simulation would not be possible using 16 order parameters without the grain tracker algorithm, as there would be no unique ID to determine which crystal orientation should be assigned to each grain.

\subsection{Grain growth in a 3D copper polycrystal}

The final demonstration of the grain tracker algorithm is in a $3 \mathrm{D}$ copper polycrystalline cube with each side having a length of $15 \mu \mathrm{m}$. The number of order parameters required to represent $3 \mathrm{D}$ grain structures is significantly larger in $3 \mathrm{D}$ than in $2 \mathrm{D}$ because of the many more interactions between grains. The 6000 initial grains are generated using a Voronoi tessellation, as shown in Fig. 6(a). The evolved microstructure is also shown. As was done in the $2 \mathrm{D}$ circle example 3.2 , we use the grain tracker information to compute the average grain volume over time (Fig. 6(b)). In addition, we compute the grain size distribution from the grain volume data taken from the grain tracker, as shown in Fig. 6(c). As has been shown in previous simulations, the grain size distribution rapidly changes until it reaches steady state. The steady state distribution deviates from the Hillert distribution [26] in a similar manner to that reported in Kamachali and Steinbach [27].

In the $3 \mathrm{D}$ simulation, 100 order parameters were required to successfully model the grain growth. The grain tracker algorithm also took a significantly larger percentage of the computation time in $3 \mathrm{D}$, accounting for $72.6 \%$ of the total computation time. While this is a significant increase over the 2D simulations, these simulations are only computationally feasible due to the algorithm. Using a non-reduced order parameter set, the 6000 grain polycrystal would require 6000 order parameters to model. The grain tracker makes this simulation possible with significantly fewer variables while still maintaining a unique ID for each grain. The number of degrees of freedom have been reduced by a factor of sixty by the grain tracker algorithm. Even with the $72.6 \%$ cost, the cost is still reduced by a factor of 40. However, ongoing work will seek to improve the performance of the algorithm in $3 \mathrm{D}$.

\section{Conclusions and Future Work}

The grain tracker is an advanced mesoscale modeling utility useful for drastically reducing the num- 


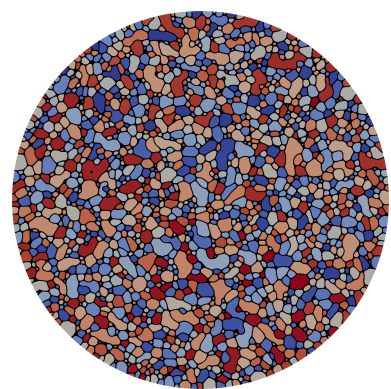

(a)

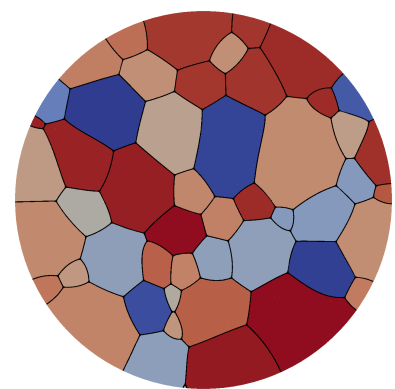

(b)

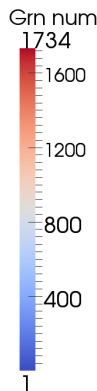

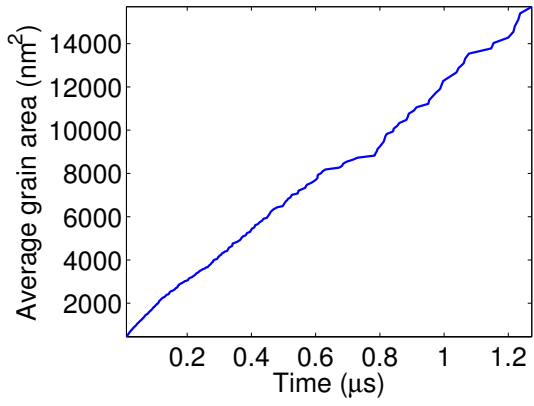

(c)

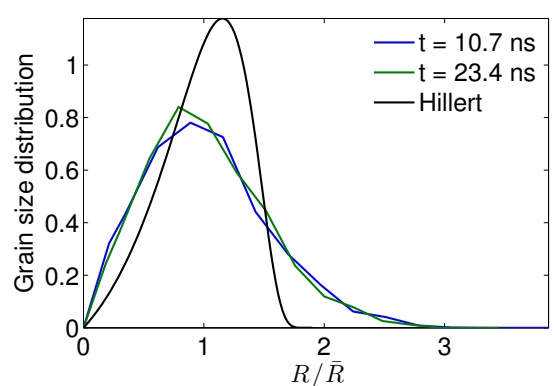

(d)

Figure 4: 2D simulations on a circular domain with zero flux boundary conditions for all order parameters in a copper polycrystal. The initial grain structure with 1723 grains, created by randomly seeding each order parameter and running for ten time steps, is show in (a). The final grain structure after $1.23 \mu \mathrm{s}$ is shown in (b). Using information collected by the algorithm, the average grain area over time is shown in (c) and the grain size distribution is shown in (d).

ber of nonlinear solution variables needed in a finite element phase-field simulation. This reduction in model sizes allows developers to execute existing models at greatly reduced simulation times on few processing cores or alternatively, enables the execution of significantly larger simulations given the same resources over current approaches. The grain tracker algorithm in MOOSE advances previous work by moving grain tracking and remapping techniques on reduced order parameter simulations into a fully-implicit Multiphysics FEM framework. The current contribution supports all time integration schemes while providing unique ids without simulation restrictions presented in previous work. The algorithm constantly monitors the locations and potential interactions of all grains in a simulation and ensures that grains represented by the same order parameters remain out of contact at all times. This is accomplished by remapping one or more order parameters of a grain nearing an interaction to another variable in the system that is not involved in the interaction while simultaneously maintain- ing a unique, unchanging identifier for every grain in the simulation. The grain tracking algorithm was designed to run completely independently of the mesoscale physics used in any particular simulation. This design enables the grain tracker to work seamlessly with advanced mesoscale simulations involving heat transfer, solid mechanics, or fission gas studies without requiring any knowledge of the physics implemented in those models nor does it require any modification of those models.

Several examples of the grain tracker algorithm working with various grain evolution models on different domain shapes under different conditions were presented and verified by comparing results (when possible) to non-reduced order parameter models. Finally, the grain tracker's impact to application run time was discussed and compared against other reduced order parameter models.

There are several modifications currently being developed to enhance the capabilities and performance of the grain tracker. Foremost, an improved simulated annealing algorithm is being in- 


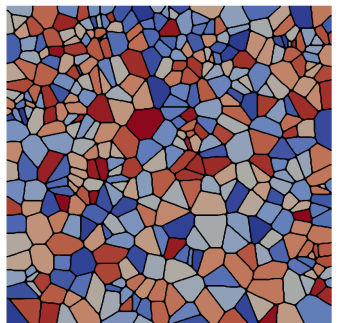

(a)

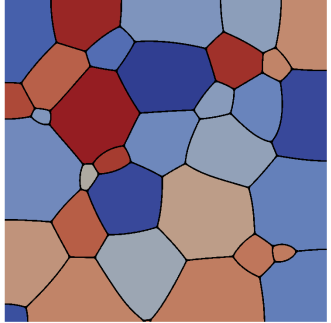

(b)

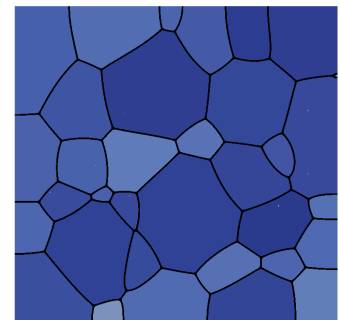

(c)

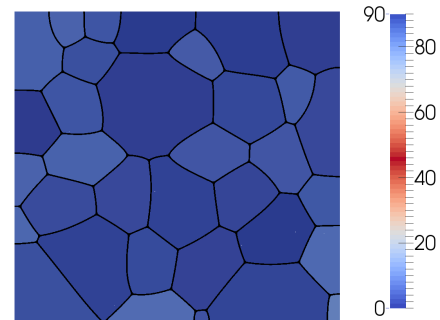

(d)

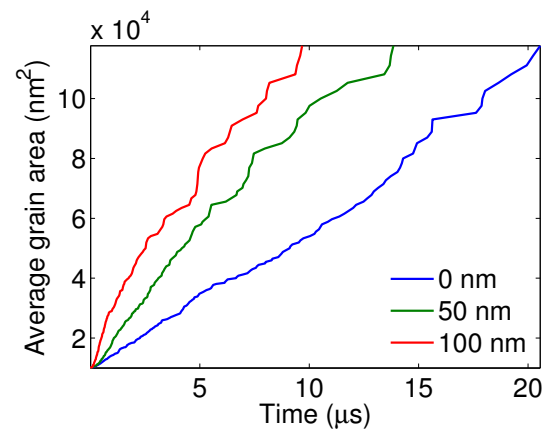

(e)

Figure 5: 2D simulations on a square domain of a 2D copper polycrystal with 400 grains under an applied load, where the initial grain structure is shown in (a). The final grain structures are shown in (b) to (d), where the grains are shaded by Euler angle. The structure with no applied strain is shown in (b), with a $50 \mathrm{~nm}$ applied load in (c), and with a $100 \mathrm{~nm}$ applied load in (d). The final texture becomes more pronounced with increasing load. The average grain area is shown over time for the three applied deformations in (e).

corporated to avoid the generation of invalid initial conditions (adjacent grains with the same order parameter) as was encountered in section 3.1. Additionally, fewer simplifications will be made to the geometry representation of the grains during the tracking and remapping phases. Instead of using enclosing spheres, exploration into polygons or other enclosing "hull" shapes will be investigated. These more complex shapes will also require more advanced collision detection methods but will further reduce the number of order parameters necessary for a given simulation. Realistic grain structures or evolution under special loading conditions can cause very anisotropic grain growth requiring a disproportionately high number of order parameters when using perfect spheres, bounding boxes or other elementary shapes to track and remap grains. These types of simulations will benefit the most from improved hull detection. Next, there are several naive proof-of-concept algorithms implemented in the merging and tracking stages that scale nonoptimally to larger problems containing several million elements. In some cases these algorithms have nearly quadratic scaling characteristics or fail to take advantage of the parallel decomposition in the problem. Additional work is planned on reducing communication by communicating only "ghost" region colorings along with the minimal spatial information to properly calculate the volume and centroid information necessary for tracking and remapping. Finally, additional research into nested grain tracking is underway to handle inclusion tracking and multiphase microstructure evolution.

\section{Acknowledgments}

This work was funded by the Department of Energy Nuclear Energy Advanced Modeling and Simulation program. This manuscript has been authored by Battelle Energy Alliance, LLC under Contract No. DE-AC07-05ID14517 with the US Department of Energy. The United States Government retains and the publisher, by accepting the article for publication, acknowledges that the United States Government retains a nonexclusive, paid-up, irrevocable, world-wide license to publish or reproduce the published form of this manuscript, or allow others to do so, for United States Government purposes. 

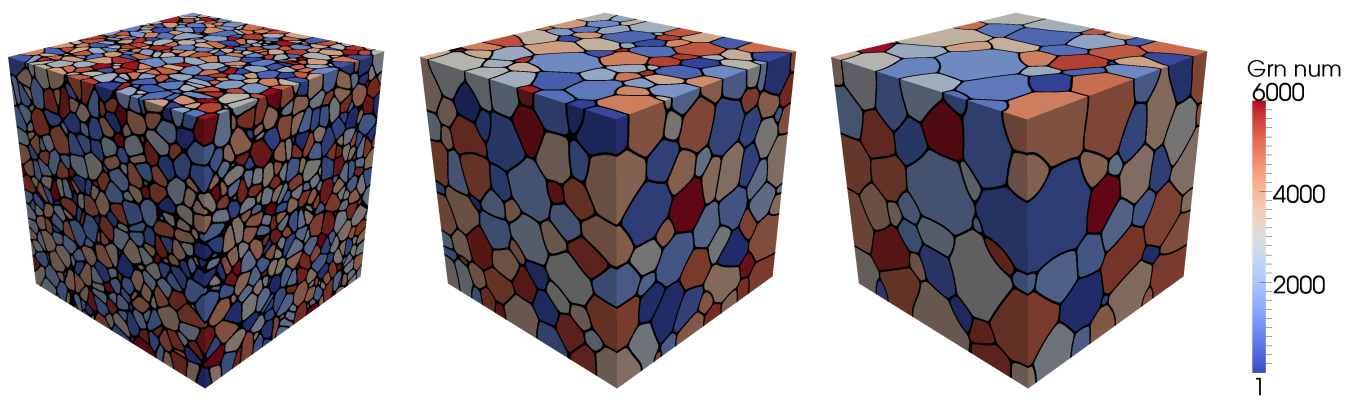

(a)

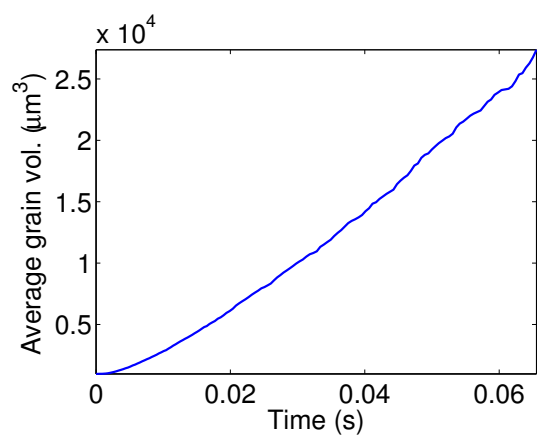

(b)

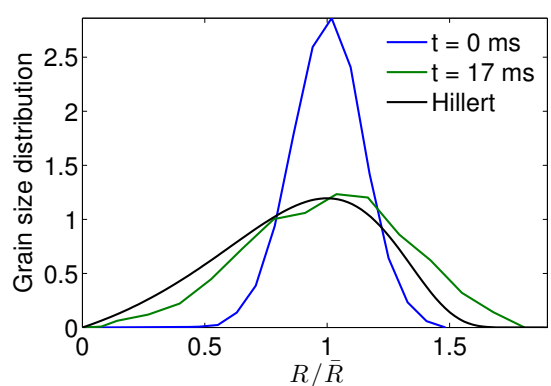

(c)

Figure 6: 3D simulations on a cubic domain of a 3D copper polycrystal with 6000 grains created with a Voronoi tessellation. The evolving grain structure is shown in (a), the the initial structure on the left, the structure after 0.03 seconds in the center and the structure after $0.065 \mathrm{~s}$ on the right. The average grain volume vs time is shown in (b) and the grain size distribution is shown in (c). The steady state distribution deviates slightly from the Hillert distribution, as expected [27].

\section{References}

[1] Anderson, M., Srolovitz, D., Grest, G., Sahni, P.. Computer-Simulation Of Grain-Growth .1. Kinetics. Acta Metallurgica 1984;32(5):783-791.

[2] Srolovitz, D., Anderson, M., Sahni, P., Grest, G.. Computer-Simulation Of Grain-Growth .2. Grain-Size Distribution, Topology, And Local Dynamics. Acta Metallurgica 1984;32(5):793-802.

[3] Frost, H., Thompson, C., Howe, C., Whang, J.. A 2-Dimensional Computer-Simulation Of CapillarityDriven Grain-Growth - Preliminary-Results. Scripta Metallurgica 1988;22(1):65-70.

[4] Moldovan, D., Wolf, D., Phillpot, S., Haslam, A.. Mesoscopic simulation of two-dimensional grain growth with anistropic grain-boundary properties. Phil Mag A 2002;82(7):1271-97.

[5] Fan, D., Chen, L.. Computer simulation of grain growth using a continuum field model. Acta mater 1997;45(2):611-622.

[6] Kim, S., Kim, D., Kim, W., Park, Y.. Computer simulations of two-dimensional and three-dimensional ideal grain growth. Phys Rev E 2006;74(6):061605.

[7] Lan, Y., Li, D., Li, Y.. A mesoscale cellular automaton model for curvature-driven grain growth. Metallurgical and Materials Transactions B 2006;37B:119-29.

[8] Zhang, X., Chen, J., Osher, S.. A multiple level set method for modeling grain boundary evolution of polycrystalline materials. Interaction MultiScale Mech 2008;1(2):178-91.

[9] Chen, J., Kotta, V., Wang, D., Moldovan, D., Wolf, D.. A variational formulation and a doublegrid method for meso-scale modeling of stressed grain growth in polycrystalline materials. Comput Methods Appl Mech Engrg 2004;193:1277-1303.

[10] Battaile, C., Counts, W., Wellman, G., Buchheit, T., Holm, E. Simulating grain growth in a deformed polycrystal by coupled finite-element and microstructure evolution modeling. Metall Mater Trans A 2007;38(A):2513-22.

[11] Zhang, L., Bartel, T., Lusk, M.T.. Parallelized hybrid monte carlo simulation of stress-induced texture evolution. Computational Materials Science 2010;48(2):419 -425 .

[12] Tonks, M., Millett, P., Cai, W., Wolf, D.. Analysis of the elastic strain energy driving force for grain boundary migration using phase field simulation. Scripta Materialia 2010;63:1049-52.

[13] Tonks, M., Millett, P.. Phase field simulations of elastic deformation-driven grain growth in $2 \mathrm{~d}$ copper polycrystals. Mat Sci Eng A 2011;528:4086-91.

[14] Moelans, N., Blanpain, B., Wollants, P.. Quantitative analysis of grain boundary properties in a generalized phase field model for grain growth in anisotropic systems. Phys Rev B 2008;78:024113.

[15] Krill, C., Chen, L.. Computer simulation of 3-d grain growth using a phase-field model. Acta Materialia 
2002;50(12):3059-3075.

[16] Gruber, J., Ma, N., Wang, Y., Rollett, A., Rohrer, G.. Sparse data structure and algorithm for the phase field method. Modelling and simulation in materials science and engineering 2006;14(7):1189.

[17] Vedantam, S., Patnaik, B.. Efficient numerical algorithm for multiphase field simulations. Physical Review E 2006;73(1):016703.

18] Vanherpe, L., Moelans, N., Blanpain, B., Vandewalle, S.. Bounding box algorithm for threedimensional phase-field simulations of microstructural evolution in polycrystalline materials. Phys Rev E 2007;76(5):056702 (pages 11)

[19] Gaston, D.R., Permann, C.J., Peterson, J.W., Slaughter, A.E., Andrš, D., Wang, Y., et al. Physics-based multiscale coupling for full core nuclear reactor simulation. Annals of Nuclear Energy 2014;

[20] Gaston, D.R., Peterson, J.W., Permann, C.J., Andrs, D., Slaughter, A.E., Miller, J.M.. Continuous integration for concurrent computational framework and application development. Journal of Open Research Software 2014;2(1):e10.

[21] Tonks, M., Gaston, D., Millett, P., Andrs, D., Talbot, P.. An object-oriented finite element framework for multiphysics phase field simulations. Comp Mat Sci 2012;51(1):20-29.

[22] Levoy, M.. Area flooding algorithms 1981;

[23] Kirk, B.S., Peterson, J.W., Stogner, R.H., Carey, G.F.. libmesh: a c++ library for parallel adaptive mesh refinement/coarsening simulations. Engineering with Computers 2006;22(3-4):237-254.

[24] Spinrad, J.P., Vijayan, G.. Worst case analysis of a graph coloring algorithm. Discrete Applied Mathematics 1985;12(1):89-92

[25] Schönfelder, B., Wolf, D., Phillpot, S., Furtkamp, M.. Molecular-dynamics method for the simulation of grain-boundary migration. Interf Sci 1997;5:245-62.

[26] Hillert, M.. On the theory of normal and abnormal grain growth. Acta metallurgica 1965;13(3):227-238.

[27] Kamachali, R.D., Steinbach, I.. 3-d phase-field simulation of grain growth: Topological analysis versus meanfield approximations. Acta Materialia 2012;60(67):2719 $-2728$ 


\section{Graphical Abstract}

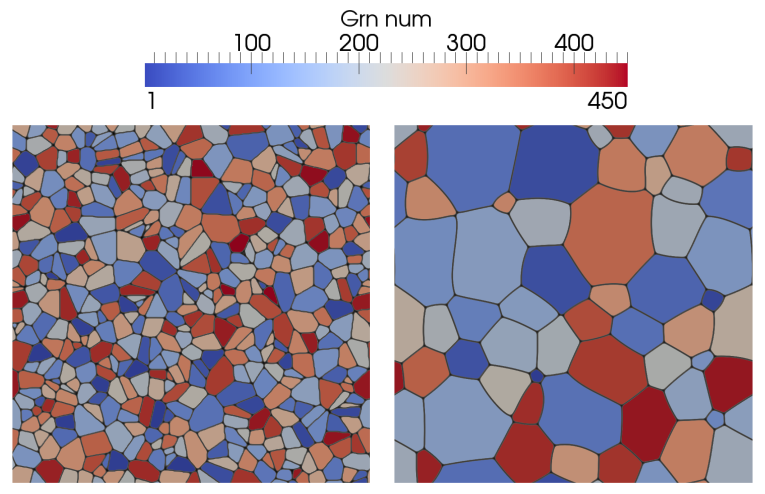

(a)

(b)

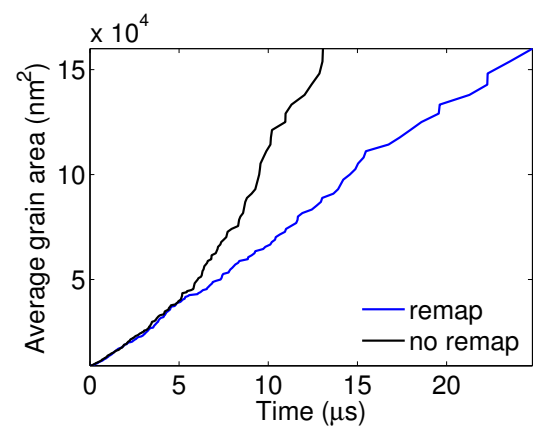

(c)

Figure 1: 2D simulation with grains spanning periodic boundary conditions. (a) initial grain structure, (b) final grain structure after $6000 \mathrm{~ns}$, (c) plot of the average grain area versus time. (a) and (b) are shaded by unique grain ID. Average grain area behaves the same for sixteen or more order parameters once the grain tracker is activated. Therefore, the model is evolving correctly (without coalescence). 